\title{
Electroluminescent optical fiber sensor for detection of a high intensity electric field
}

\author{
Tadeusz Pustelny* \\ Silesian University of Technology, Department of Optoelectronics, 2 Krzywoustego St., 44-100 Gliwice, Poland
}

Received March 13, 2020; accepted March 26, 2020; published March 31, 2020

\begin{abstract}
On-line testing of high-power electromagnetic devices is one of the most important problems of modern industrial metrology. In the paper, the results of experimental investigations of an electric field optical fiber sensor based on electroluminescent phenomena are presented. The electroluminescent effect is observed in some composite semiconductors, among others in zinc sulfide $\mathrm{ZnS}$ crystals. In our investigations, the used $\mathrm{ZnS}$ crystal was doped with copper $\mathrm{Cu}$ atoms as activators. The concentration of an activator in the $\mathrm{ZnS}$ crystal was about $5 \cdot 10^{-4}[\mathrm{~g} / \mathrm{g}]$. According to the plans of investigations of the elaborated electroluminescent sensor, spectral properties as well as the intensity of light emission in the sinusoidal alternating electric field were tested.
\end{abstract}

Fiber optic sensors are increasingly used in metrological devices in both industry and science. The main advantage of fiber optic sensors is their insensitivity to interference of intense electromagnetic fields. Fiber optic sensors and optical channels are made of highquality insulating dielectric materials, without metal elements. In high voltage transformers, the measurements of electric field strength provide a lot of interesting and important information about their operating conditions and conditions at risk of damage $[1 \div 2]$. The idea of a contactless method for the measurement of electric field intensity is based on the influence of an electric field on the optical properties of $\mathrm{ZnS}: \mathrm{Cu}$. As a result of the electric field action on this electroluminophore, light emission from them is observed [3]. In electroluminophores, the intensity and spectrum of luminescent light is the function of electric field intensity [4].

There are two types of electroluminescence phenomena

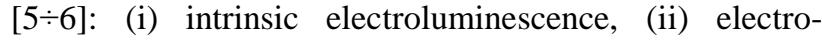
luminescence by electric carrier injection.

Most of the known electroluminophores are based on semiconductors of the II-VI compounds [7]. Electroluminophores are prepared similarly to activated crystalline photoluminophores [5]. The concentration of activator materials in electroluminophores is about $10 \div 100$ times higher than that in photoluminophores. Electroluminophores should not have too many defects that disturb the acceleration of electrons excited in the centres of luminescence [7].

\footnotetext{
*E-mail: Tadeusz.Pustelny@polsl.pl
}

The electroluminescent $\mathrm{ZnS}$ has been obtained by doping the based material with proper donor activators [8]. Electroluminescent materials may be also obtained by mixing $\mathrm{ZnS}$ powder with metal powder. From literature it appears that experimental relation between the intensity of electroluminescent light and electric field intensity may be presented often in the following form $[4 \div 5]$ :

$$
I(E)=A_{o} \exp \left(-\mathrm{bE}^{-1}\right)
$$

where: I - the intensity of electroluminescence; $\mathrm{A}_{0}, \mathrm{~b}-$ the constant values, dependent on the kind of luminophore; $\mathrm{E}$ - the intensity of the electric field in the electroluminophore.

For the majority of electroluminophores, the relation (1) is correct in some orders of the electric field intensity.

When an electroluminophore is directly placed in constant electric field $\mathrm{E}$, the light emission has the nature of a fast fading impulse. This result can be explained by the dielectric polarisation effect in phosphor [8].

The electroluminescent effect obtained by the injection of electric charges is observed if the phosphor is in electric contact with metal electrodes, to which voltage is connected or if aluminophore is used in the $p-n$ junction [9]. The use of this effect in sensors to control the operating status of electrical transformers is practically impossible due to the need to supply electrical power to the sensor.

In the papers $[8,10]$ it is shown that in thin plates of $\mathrm{ZnS}$ with adequate activators aphotoelectroluminescent signal may be observed. These effects result from simultaneous application of an electric field and illumination to the crystal $[11 \div 12]$. It is practically impossible to use this effect in sensors of an electric field in power machines (transformers).

After our preliminary tests, we chose some $\mathrm{ZnS}$ luminophores for our investigations and for their future application in electric field intensity sensors. In our investigations, the electroluminescent sensor based on zinc sulphide doped by copper $\mathrm{ZnS}-\mathrm{Cu}$ at a concentration of activator $5 \cdot 10^{-4}[\mathrm{~g} / \mathrm{g}]$ was tested. The presented sensor had the best performance properties: relatively high emission of electromagnetic radiation and the best mechanical properties. For the tests, electroluminophores were prepared in the form of electroluminescent cells. 
The construction of the electroluminescent cell is presented in Fig.1.

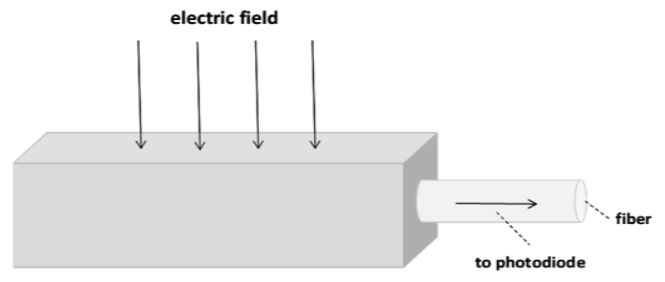

Fig. 1. Electric field sensor in the form of an electroluminescent cell.

The electroluminescent measuring cells were made in the form of a small cuboid (with a thickness of $400 \mu \mathrm{m}$ and several $\mathrm{mm}$ dimensions). For reasons of measurements security, the phosphor was placed between two plates of a mica insulator. The electric and optical properties of the phosphor placed in an electrical capacitor were tested. We used optical fibers, which were elaborated in the Fiber Optic Laboratory of Maria Curie-Sklodowska University in Lublin. The applied optical fiber had $300 \mu \mathrm{m}$ in fiber core diameter, a total diameter of $400 \mu \mathrm{m}$, and 0.43 numerical aperture.

The experimental stand for the investigation of sensor optical properties is presented in Fig.2.

The alternating electric voltage was generated by an autotransformer. The electric field of high intensity was formed in a capacitor, where electroluminescent cells were placed inside.

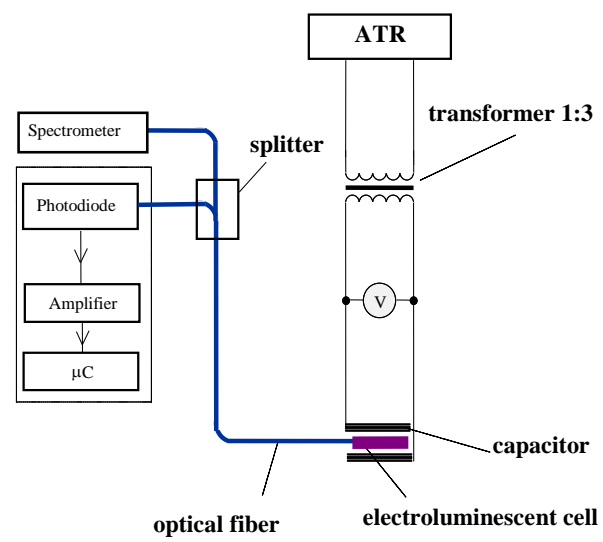

Fig. 2. The stand for testing the electroluminescent sensor in high intensity electric fields.

From the electric cell, the electroluminescent light was led to the photodetector by means of an optical fiber. Next, the electric signal after amplification was directed to an $\mathrm{A} / \mathrm{C}$ converter and next was analyzed in the computer. In the set-up, an A/D converter of the 1600 DAS type (Keithley MetraByte LTD) was used. The stand allowed, additionally, investigating the same electroluminescent signal by a spectrometer (spectrometer type 14.1 WOCAD). A high sensitivity photodiode FLM 3B was used for detecting the electroluminescent signal. In alternating electric fields, the electroluminophores shine in the form of brightness waves [5]. The frequency of brightness waves is two times greater than the frequency of an electric field. The shape of brightness waves in time depends on the kind of luminophore. For applying $\mathrm{ZnS}-\mathrm{Cu}$, the brightness waves had the sinusoidal shape (Fig.3).

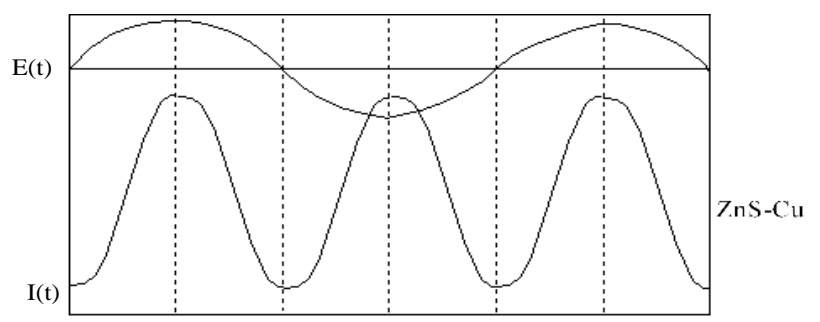

Fig. 3. Brightness waves in $\mathrm{ZnS}-\mathrm{Cu}\left(5 \cdot 10^{-4} \mathrm{~g} / \mathrm{g}\right)$.

Figure 4 shows the spectrum of the electroluminescent signal obtained for $\mathrm{ZnS}-\mathrm{Cu}$ (with $\mathrm{Cu}$ concentration: $\mathrm{n}_{\mathrm{Cu}}=$ $5 \cdot 10^{-4} \mathrm{~g} / \mathrm{g}$ ) at the electric field intensity $\mathrm{E}=1200 \mathrm{~V} / \mathrm{mm}$. In the cell, the optical fiber with $300 \mu \mathrm{m}$ optical core diameter was used. The spectrum of the electroluminescent signal is relatively wide in the range $500 \div 600 \mathrm{~nm}$.

In Figure 5, the relations between the intensity of the electric field $\mathrm{E}$ and the intensity of the emitted light $I=f(E)$ are presented (where in Figs. $3 \div 5$, the intensities of electroluminescence are presented in arbitrary units).

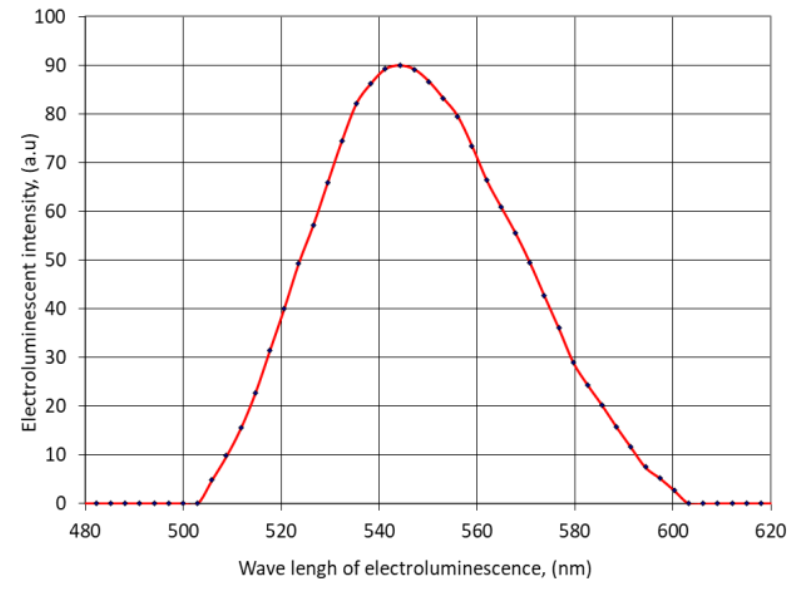

Fig. 4. Spectral characteristics of applying electroluminophore $\mathrm{ZnS}-\mathrm{Cu}$

The characteristics presented in Fig. 5 shows that the determination accuracy of electroluminescent intensity depends very strongly on the intensity of an electric field. For electric field intensity up to several hundred $\mathrm{V} / \mathrm{mm}$ the accuracy is rather small - the order of a few percent. 
For electric field intensity above $1000 \mathrm{~V} / \mathrm{mm}$ the presented measurement system allows obtaining the accuracy results o of the order of $1 \%$ (for an even higher electric field the accuracy may be even better).

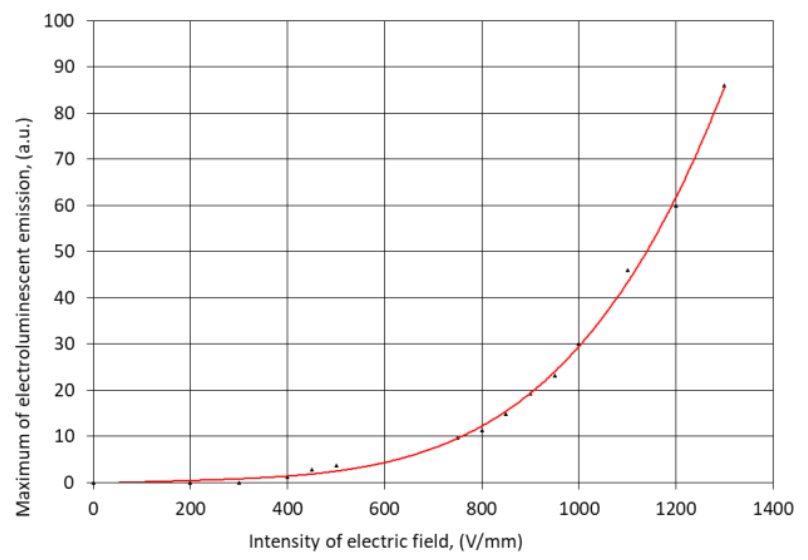

Fig. 5. Electroluminescence intensity as a function of the electric field.

Figure 6 shows the same relation but in the coordinate system: $\ln \left(\mathrm{I} / \mathrm{I}_{\mathrm{o}}\right)=\mathrm{f}(1 / \mathrm{E})$. It may be stated that the experimental relation between the intensity of electroluminescent light and the intensity of an electric field is in good agreement with the relation predicted theoretically and described by Eq. (1).

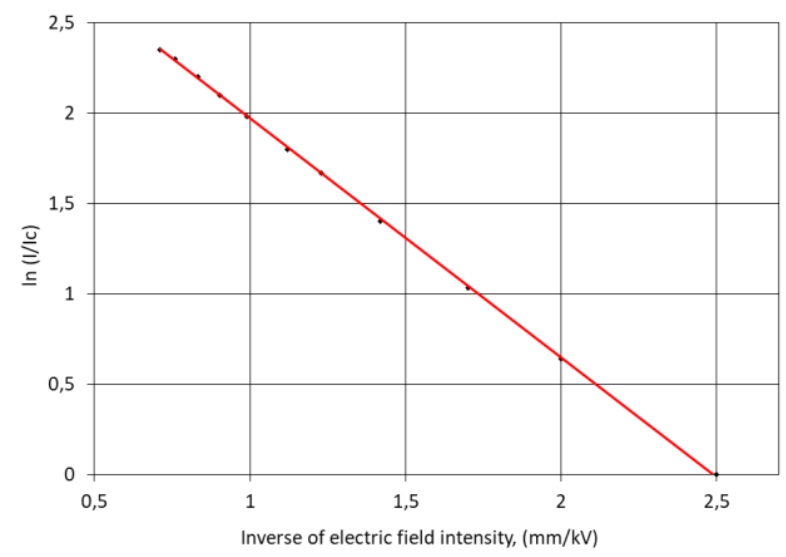

Fig. 6. The electroluminescence intensity depending on the electric field, presented as the function: $\ln \left(\mathrm{I} / \mathrm{I}_{\mathrm{o}}\right)=\mathrm{f}(1 / \mathrm{E})$.

Based on $\mathrm{ZnS}-\mathrm{Cu}$, a fiber optic electric field intensity sensor was elaborated. The tests of the fiber optic sensor for measuring high intensity electric fields (up to $2 \mathrm{kV} /$ $\mathrm{mm})$ were presented. The optical properties of selected $\mathrm{ZnS}-\mathrm{Cu}$ with a concentration of activators $\sim 5 \cdot 10^{-4} \mathrm{~g} / \mathrm{g}$ were examined. Our sensor belongs to a group of optical fiber intensity sensors [1]. For this reason, it is very important to assure good and stable coupling between the optical fiber and electroluminophore, as well as between the optical fiber and a photodetector. We could observe that the relation between the intensity of electroluminescent emission of light and electric field intensity is of an exponential character (according to Eq. (1) and Fig. 6). The exponential investigations confirm that the sensitivity of our system is much higher for a higher electric field intensity. Because of this, the electroluminescent sensors are aimed, first of all, at the measurement of high electric field intensity. For a weak electric field, the obtained results are very uncertain. The fiber optic sensor of electric intensity is of small dimensions. Its construction is not complex. The optical parts of sensors e.g. the electroluminescent cell and optical fiber channel are made of non-metallic elements of high resistivity. For all these reasons, there is a chance that the electroluminescent fiber optic sensors as well as the optical fiber magnetic field sensors $[13 \div 14]$ will find use in high voltage electrical systems for controlling how they work.

The author would like to thank very much the Riedelde Haen AG for providing electroluminophores. The author would like to thank to MSc. W. Trzaskalik for the participation and help in the experimental investigations.

\section{References}

[1] K.T.V. Grattan, Fiber Optic Fluorescence Thermometry (Chapman and Hall, London 1996).

[2] K. Kyuma, S. Tai, T. Sawada, J. Quntum. Electron. 73(3), 363 (1997).

[3] A. Brief, J. Chem. Educ. 88(6), 731 (2011).

[4] T. Pustelny, B. Pustelny, Opto-Electronics Rev.10(3), 193 (2002).

[5] A.Wrzesinska, Photo- and electroluminophore (PWN Press, Wroclaw 1988); in Polish.

[6] K.A. Franz, W.G. Kehr, Luminescent Materials, Ullmans Encyclopedie of Industral Chemistry (Wiley-VCH, Veinhen 2008).

[7] A.G. Milnes, Deep Impurities in Semiconductors (WilleyInterscience Public., Toronto 1993).

[8] M. Aven, J.S. Prener, Physics and Chemistry of II-VI Compounds (North-Holland Publishing Company, Amsterdam 1993).

[9] P.K. Cheo, Fiber Optics Devices and Systems (Prentice-Hall 1985).

[10] D. Randall, Fluorescence and Phosphorescence (Grown, Oxford 2007).

[11] M. Koen, Photoconductivity of Semiconductors (Ed. Parks, New York 1996).

[12] K.R. Murohy, C.A. Stedman, Annal. Methods 6(3), 658, (2014)

[13] T. Pustelny, K. Barczak, K. Gut, J. Wojcik, Opt. Appl. 34(4), 531 (2004).

[14] K. Barczak, T. Pustelny, D. Dorosz, J. Dorosz, Europ. Phys. J. 154, 11 (2008). 